\title{
Ungarndeutsche Kinderliteratur: Josef Michaelis‘ Zauberhut
}

\author{
Kerekes, Gábor \\ ELTE Bölcsészettudományi Kar Germanisztikai Intézet Német Nyelvü Irodalmak Tanszéke
}

\begin{abstract}
Absztrakt
The study deals with the most succesful book of the Germans in Hungary, with Josef Michaelis' Zauberhut [Magic Hat], a book that contains poems and tales for children. It is presented in severals contexts, so in the context of the Hungarian bookmarket as well as in respect of its birth and the international literary context of some poems.
\end{abstract}

Keywords: children's literature, literature of the Germans of Hungary, Josef Michaelis

\section{Der Autor}

Josef Michaelis ist der erfolgreichste ungarndeutsche Autor der Gegenwart. Er erblickte als Michelisz József 1955 als Sohn einer ungarndeutschen Mittelbauernfamilie in Schomberg/Somberek in der Branau/Baranya das Licht der Welt. Nach Schule und Mittelschule studierte er an der Hochschule für Lehrerbildung in Frankenstadt/Baja Deutsche Sprache und Literatur, danach Geschichte an der Janus-Pannonius-Universität in Fünfkirchen. 1999 absolvierte er einen Fortbildungslehrgang an der ELTE und wurde Lehrplanexperte für Deutsch als Nationalitätensprache. Seit 1977 arbeitete er als Lehrer für Deutsch und Geschichte in Willand/Villány (Márkus, 2009) und war seit 1991 stellvertretender Schulleiter in der Grundschule (Kerekes, 2015). Seit 2020 ist er in Rente.

$\mathrm{Zu}$ schreiben begann er zunächst in ungarischer Sprache, den Weg zum deutschsprachigen Schreiben ebnete ihm der Fünfkirchener Hochschulgermanist Béla Szende, wobei eine wichtige Initialzündung ein Fortbildungskurs in Potsdam war, zu dessen literarischem Ertrag ein Gedicht (Mädchen aus Potsdam) gehört, das später in seinem Gedichtband Sturmvolle Zeiten erschien. Eine besonders wichtige Person war für Michaelis der als Lektor tätige Jochen Haufe (1928-2014), an den sich Michaelis mit viel Liebe erinnert: "Jochen Haufe war aber die Person, die mich in eine andere Welt und Richtung der Dichtkunst einführte, [...] Jochen war für mich ein ,literarischer Wegweiser' auf den unwegsamen und unbekannten Wegen der 
modernen Dichtung. Seine Meinung zu den Werken habe ich immer akzeptiert [...]. Und von ihm konnte man in jeder Hinsicht sehr viel lernen." Eine weitere, für die Formung seines literarischen Schaffens wichtige Person war der 2007 verstorbene Helmut Rudolf, über den der Dichter sagt: „er war jahrelang treuer Lektor meiner Gedichte“.2 Nachdem sich Michaelis dem deutschsprachigen Schreiben zugewandt hatte, wurde sein Talent offenkundig und er war bald mit seinen Gedichten in den verschiedenen Publikationen der Ungarndeutschen (Neue Zeitung, Signale, Deutscher Kalender, diverse Anthologien) im In- und Ausland zu lesen (Szabó, 2001).

Die Bedeutung, die er im Laufe der Zeit für die Volksgruppe und ihre Literatur gewonnen hat, unterstreicht auch die Tatsache, dass 1992 mit seinem Gedichtband Sturmvolle Zeiten die Veröffentlichungsreihe der VUdAK-Bücher, der bis heute einzigen Reihe, die sich der Publikation ungarndeutscher Literatur widmet, ihren Auftakt nahm.

Selbständige Bände besitzen nicht viele ungarndeutsche Autoren, Josef Michaelis kann aber über die erwähnte erste VUdAK-Veröffentlichung sowie den hier zur Betrachtung im Mittelpunkt stehenden Band hinaus auch noch auf die Gedichtsammlungen Treibsand (2004) und Symbiose (2015) sowie die zweisprachige Veröffentlichung Der verlorene Schatz / Az elveszett kincs (2008), die mit Märchen und Sagen aufwartet, verweisen.

Seine Werke zeugen nicht nur von einer tiefen Verbundenheit mit der Sagen- und Märchenwelt der Ungarndeutschen, sondern auch mit der Geschichte und dem Schicksal der Volksgruppe. Josef Michaelis hat sich zu keiner Zeit dem ungarndeutschen Schicksal gegenüber indifferent gezeigt, sondern mit mehreren seiner Gedichte eindrucksvolle, intelligente und den Lesern ob ihres Wahrheitsgehaltes bestürzende Werke verfasst, die sich unauslöschlich in die Geschichte der ungarndeutschen Literatur eingeschrieben haben. So kann man zum Beispiel die Gedichte Agonie, Die Räder rattern, Branauer Schwäbin, Mein Ungarndeutschtum (um nur einige dieser Werke zu erwähnen) durchaus als Klassiker der ungarndeutschen Literatur bezeichnen. Er hat immer wieder seine Meinung zu grundsätzlichen Fragen des öffentlichen Lebens und der Politik artikuliert, darüber hinaus spannt sich der Bogen seiner Lyrik über weite historische Perspektiven zu Erinnerungs- und Stimmungsbildern besuchter bzw. bekannter Orte sowie zu intim-sensiblen Gefühlsregungen sowohl emotionaler als auch philosophischer Art, die auf zarteste Weise eingefangen worden sind. Sein Gedicht Mein Ungarndeutschtum ist ein die Identität der Ungarndeutschen bei aller Verbundenheit zur deutschen Sprache und mit dem Erbe der aus Deutschland stammenden Vorfahren als eine auch sehr deutlich auf den in der ungarischen Heimat entstandenen neuen Traditionen und Sitten fußende Selbstbestimmung zeichnendes Werk, das die einzigartigen Züge der Volksgruppe vor Augen führt.

\footnotetext{
${ }^{1}$ Mitteilung von Josef Michaelis in einer E-Mail an mich vom 20. Mai 2020.

${ }^{2}$ Mitteilung von Josef Michaelis in einer E-Mail an mich vom 11. Juni 2020.
} 
Ein besonders wichtiges Element der ungarndeutschen Identität von Josef Michaelis ist die Sprache, die Muttersprache, mit der die deutsche Sprache gemeint ist, die in einer Reihe seiner Gedichte als Thema eine zentrale Rolle spielt. Zu den Umständen des Sprachgebrauchs der Ungarndeutschen vgl. Knipf-Komlósi (2011), und Knipf-Komlósi \& Müller (2019). Zu der Sprachlandschaft (linguistic landscape), die die Ungarndeutschen umgeben s. und Knipf-Komlósi \& Müller (2018) sowie Müller (2013).

Das lyrische Werk Michaelis' ist über die thematische Vielseitigkeit hinaus auch gekennzeichnet von einem formalen Reichtum. Neben Gedichten, die in ihrer klaren, abgeklärt-gleichmäßigen Form den jahrhundertealten Dichtungstraditionen der deutschsprachigen Literatur entsprechen, finden sich in seinem Lebenswerk auch eine Reihe von Gedichten in freier Form, freiem Rhythmus und freien Strophen, dann auch spielerische, graphische Elemente aufweisende Bildgedichte, mit unübersehbaren Anleihen an die konkrete Poesie. Besonders bemerkenswert ist dabei, wie sich in jeder formalen Gestaltungsweise ansprechende Gedichte finden. Überhaupt ist es ein Zug von Michaelis, sich nicht in unverständlich verklausuliert-nebelige Formulierungen zurückzuziehen, sondern ein Dichter für Leser zu bleiben, ohne dabei in plakativ-plumpe Direktheit abzurutschen.

Seinen literarischen Rang verdeutlicht auch der Umstand der Übertragungen seiner Gedichte ins Ungarische, Englische und Slowakische.

\section{Das Buch}

\section{Veröffentlichung und Auflagenhöhe}

Das für Kinder gedachte Buch Zauberhut erlebte bis heute insgesamt fünf Auflagen, drei ausschließlich in deutscher Sprache (1991, 1994 und erweitert 2001) und zwei weitere Auflagen zweisprachig als Zauberhut / Varázscilinder (2005 und 2008), womit es das am häufigsten veröffentlichte Buch der ungarndeutschen Literatur bis dato ist. Die Erstausgabe und jene von 1994 besitzen 102 Seiten, während die 3. Ausgabe von 2001 insgesamt 106 Seiten umfasst. In dieser letzteren befinden sich neu nunmehr zusätzlich auf S. 41-42 das Gedicht Drachenabenteuer und auf S. 47-48 Die Nik(olaus)maus, weshalb der Band auch als „erweiterte Ausgabe“ bezeichnet wurde. Herausgegeben wurde die erste Ausgabe von dem ungarischen Schulbuchverlag Tankönyvkiadó, die zweite Auflage von dem Verband der Ungarndeutschen und die dritte von der Landesselbstverwaltung der Ungarndeutschen, was deutlich die Veränderungen ausdrückt, die sich in den Jahren nach der politischen Wende in Ungarn abgespielt haben.

Mit einer Auflagenzahl von insgesamt 8.000 Tausend Exemplaren (1. Auflage: 2.000 Exemplare; 2. Auflage: 3.000 Exemplare; 3. Auflage: 3.000 Exemplare) hat das Buch eine bemerkenswert hohe Auflagenzahl erreicht, die 
- wenn man die beiden zweisprachigen Ausgaben noch hinzurechnet - um weitere 4.100 Exemplare zunimmt. Diese 12.100 Exemplare stellen eine beachtliche Zahl an Büchern dar, vor allem wenn man bedenkt, dass in Ungarn heutzutage als nur sehr selten anzutreffende Spitzenwerte im Buchhandel Auflagen von 30.000 Exemplaren gewertet werden, die aber auch nur von ausgesprochen dem Bereich der Unterhaltungs- und Trivialliteratur angehörenden Werken erreicht werden, wie jene des auch unter dem Pseudonym L.L.Lawrence erscheinenden Bücher von László L. Lőrincz oder die Verschwörungsthriller von Dan Brown (STAT 2015). 2018 etwa gab es in Ungarn nur ca. 20 Bücher, die überhaupt eine Auflagenzahl von über 10 Tausend Exemplaren erreichten. ${ }^{3}$ Setzt man dies nun in Relation zu dem Zauberhut und seinen Auflagenzahlen, so darf man natürlich nicht übersehen, dass das Michaelissche Buch für die Kinder einer nationalen Minderheit in Ungarn gedacht ist, die - grob gerechnet - etwa aus 200.000 Angehörigen besteht. Zu den statistischen Angaben der deutschen Minderheit in Ungarn vgl. Knipf-Komlósi \& Müller (2018), zum Sprachaneignungsprozess von Kindern der deutschen Minderheit in Ungarn vgl. Márkus \& Gölcz (2018). (Als Gegenprobe, um diesen Umstand zu unterstreichen: Den 1982 erschienenen und damals sehr begehrten, so gut wie gar nicht zu erhaltenden Doppelband mit den Theaterstücken Equus und Amadeus des damals in Ungarn wegen der Aufführungen dieser Werke am Vígszínház und Pesti Színház äußerst populären Peter Shaffer, der in etwa die Auflagenhöhe von Zauberhut aufweist und bis heute nicht noch einmal auf Ungarisch veröffentlicht worden ist, kann man heute ohne Weiteres auf beinahe allen der obengenannten Portale erhalten.) Natürlich sollte man nicht vergessen, dass das Buch auch kleine Leser unter das Deutsche erlernenden, nicht zur ungarndeutschen Minderheit gehörenden ungarischen Kindern gefunden haben mag, doch auch dann noch ist die Diskrepanz zwischen dem rund 10 Millionen Menschen ausmachenden Markt für ungarische Bücher und den 200.000 Personen der Ungarndeutschen immens, und diese Zahlen zueinander in Relation setzend wird erst wirklich ersichtlich, wie hoch die Auflagenzahlen von Zauberhut sind und welche Bedeutung das Buch für das Ungarndeutschtum besitzt. Trotz dieser hohen Auflagenzahl ist das Buch im antiquarischen Bereich (auch online unter antikva.hu; antikvarium.hu; bookline.hu; regikonyvek.hu; vatera.hu etc.) so gut wie gar nicht zu erhalten, und wenn ein Exemplar angeboten wird, so ist es schnell vergriffen - was deutlich zeigt, wie stark die emotionale Beziehung der Besitzer von Zauberhut zu ihrem Exemplar des Kinderbuches ist. Die lobenden Worte von Helmut Rudolf (1991) nach dem Erscheinen der ersten Auflage sowie jene von Ingmar Brantsch (1994) nach der Veröffentlichung der zweiten Auflage haben sich im Laufe der Jahrzehnte als vollkommen berechtigt erwiesen.

${ }^{3}$ https://forbes.hu/uzlet/mutatjuk-igy-olvas-a-magyar-de-hogy-keresnek-idehaza-az-irok/ (15. Mai 2020) 
Diese Popularität des Zauberhut unterstreicht nicht nur, wie gut Josef Michaelis die Sprache und die Vorstellungswelt der Kinder getroffen hat was an sich schon eine nicht zu unterschätzende Leistung ist, denn gerade sie sind insofern besonders ehrliche und kritische Leser, als dass sie sich von Texten und Verfassern, die sie nicht ernst nehmen, schnell abwenden. Vielmehr besteht die Stärke und so auch die Grundlage des Erfolgs des Zauberhut gerade darin, dass Michaelis den Kindern die Achtung und Empathie entgegenbringt, die sie verdienen. Seine kleinen Leser spüren das und sind ihm auch dementsprechend dankbar.

\subsection{Die Entstehung des Buches}

Solch eine auf die Bedürfnisse und Kenntnisse der Kinder eingehende Lektüre entsteht nicht durch Zufall. Durch die eigene tagtägliche Arbeit an der Schule war Michaelis zu jeder Zeit in Kontakt mit seiner potenziellen Lesergruppe, für die es in der ungarndeutschen Literatur durchaus schon Texte und Publikationen gab, allen voran die Kinderanthologie Igele-Bigele aus dem Jahr 1980. Doch waren viele der für Kinder verfassten Texte problematisch. Michaelis erkannte: „Die Texte der damaligen Schulbücher für Nationalitätenschüler waren sprachlich vielmal schwer, manchmal politisch gesinnt und viele Geschichten hatten mit dem Ungarndeutschtum, mit unseren Traditionen nichts zu tun." Bei Lesungen ungarndeutscher Autoren an Schulen wurde deutlich, welche Schwierigkeiten die Schüler mit dem Verständnis von einer Reihe für sie geschriebenen Texten hatten, da weder die Sprache noch die dargestellten Themen für sie nachvollziehbar waren. So kam Josef Michaelis zu dem Entschluss, selber für Kinder zu schreiben, wobei er sich als auslösendes Moment an eine Lesung in Tarian/Tarján an der Grundschule erinnert, wo die erwähnten Probleme deutlich zu Tage traten. Die damalige Deutschlehrerin an der Schule, Theresia Klinger, die selbst Verfasserin zahlreicher Deutschbücher war, empfahl den Anwesenden, speziell für Kinder zu schreiben. Michaelis beherzigte diesen Vorschlag und begann seine den Kindern gewidmete literarische Tätigkeit, die auch heute noch, beinahe dreißig Jahre später nicht abgeschlossen ist, wie das sein Kindergedicht $R e$ genbogen aus dem Jahr 2018 unterstreicht.

Als grundlegend wichtig ist die Bestrebung hervorzuheben, wie Michaelis die Vermittlung, die Förderung der deutschen Sprache als eine Aufgabe, ja als eine Mission ansieht, der er sich nicht verschließen kann. Die tiefe Verbundenheit zur deutschen Sprache möchte er auch in seinen Lesern erwecken. Über sein Verhältnis zur deutschen Sprache schrieb er:

Meine Muttersprache ist eine fränkische Mischmundart, die ich von meinen Eltern als Säugling in meine Wiege als größtes Geschenk meines Lebens bekommen habe. Bis zu meinem sechsten Lebensjahr beherrschte ich

${ }^{4}$ Mitteilung von Josef Michaelis in einer E-Mail an mich vom 4. Mai 2020. 
nur diesen eigenartigen Dialekt. In dieser Mundart wuchs ich auf, in dieser sprach ich mit meiner Urgroßmutter, mit meinen Großmüttern, Großvätern, mit Verwandten und mit meinen Eltern bis zu ihrem Tod. In dieser Mundart erklangen die ersten Kinderreime und Kinderlieder aus dem Mund meiner lieben Mutter.

Den Kindergarten besuchte ich nicht, so musste ich eigentlich die ungarische Sprache in der Grundschule erlernen. Es war gar nicht so leicht, aber nach zwei-drei Monaten sprach ich schon fast fehlerlos ungarisch. In der Dorfschule studierte ich auch Deutsch, und dort erfuhr ich bald, dass die Hochsprache (Standardsprache?) nicht immer so klingt wie zu Hause meine Mundart, aber sie half mir immer - damals im Kindesalter, später als Student und Lehrer, und auch heute noch als Autor. Ohne meine Mundart, meine Muttersprache wäre ich nur ein Bettler auf dieser Welt (ebenda). ${ }^{5}$

Die Texte für den Zauberhut entstanden im Laufe der Zeit, wobei die Schüler vom Michaelis die jeweils entstandenen neuen Texte zuerst kennenlernten. Ihre Reaktionen und Bemerkungen halfen dabei, die Texte immer weiter zu verfeinern, dem sprachlichen Niveau und der allgemeinen Sichtweise der Kinder anzunähern.

\subsection{Der Aufbau des Buches}

Das Buch besteht aus drei Teilen, die Reimschnitzerei, Jahreszeiten und Zauberhut betitelt sind.

\subsubsection{Reimschnitzerei}

Der erste Teil des Buches enthält - ebenso wie auch Teil zwei - nur Gedichte, die sprachlich einfach gehalten sind, damit sie für die Kinder verständlich sind. Zugleich wird auf diese Weise in den Texten den Lesern unverkrampft ein neuer Wortschatz vermittelt, etwa im Auftaktgedicht Reimschnitzerei durch die Aufzählung von Berufen und den Tätigkeiten, die die einzelnen Vertreter dieser Professionen ausüben (Michaelis 1994, p. 13, im Weiteren: $\mathrm{ZH})$ :

\footnotetext{
${ }^{5}$ Näheres zur Einstellung der Vertreter der älteren Generation der Ungarndeutschen zur deutschen Sprache findet man in Miskei \& Müller (2020, pp. 327-343).
} 


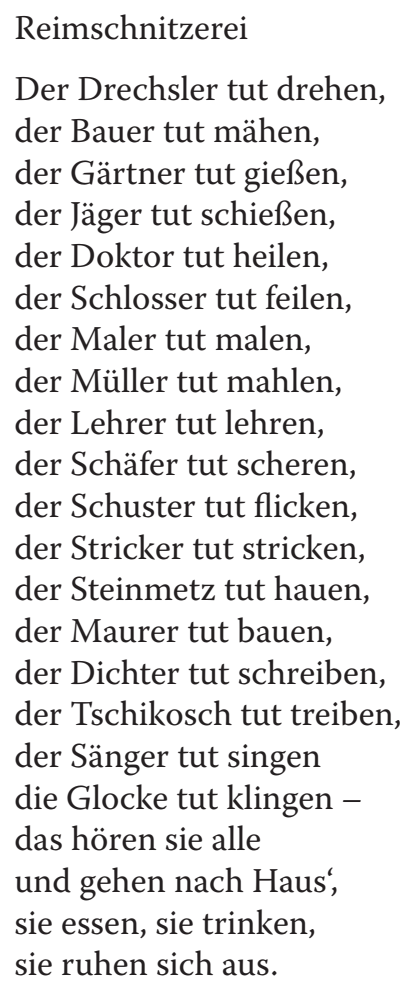

Ähnlich werden einige Seiten weiter auch im Gedicht Wenn der Schneider... Berufe (Schneider, Schuster, Schmied, Maurer, Maler, Bäcker, Fleischer) von Michaelis vorgestellt. In beiden Fällen ist der Tonfall ein freundlich-beruhigender, dem auch Ironie nicht fremd ist, was dem Umfeld des Kinderbuches angemessen ist. Diese Art der Aufzählung von Berufen und Tätigkeiten scheint vielleicht auf den ersten Blick als ein nur für die Kinderliteratur zweckmäßiges Schema zu sein, doch findet es sich in der modernen deutschsprachigen Lyrik auch an anderer Stelle: Der als Lyriker seine Laufbahn beginnende österreichische Autor Thomas Bernhard - der hinsichtlich des Zielpublikums und der Thematik seiner Werke nichts mit Kindern zu tun hatte, sondern in den meisten seiner Werke die Vergänglichkeit und die scheinbare Sinnlosigkeit des Lebens bedauerte - nutzte in seinem eine ganz andere Zielsetzung besitzenden Gedicht Ahnenkult aus dem Jahr 1977 das gleiche aufzählende Verfahren wie Michaelis, und auch bei ihm erscheint der Dichter im Text (Bernhard 1977, pp. 21-23): 
Es steigt der Steiger

bis er nicht mehr steigt

es schweigt der Schweiger

bis er nicht mehr schweigt

es lacht der Lacher

bis er nicht mehr lacht

es macht der Macher

bis er nicht mehr macht

es kocht der Kocher

bis er nicht mehr kocht

es locht der Locher

bis er nicht mehr locht

es tötet der Töter

bis er nicht mehr tötet

es flötet er Flöter

bis er nicht mehr flötet

es taucht der Taucher

bis er nicht mehr taucht

es raucht der Raucher

bis er nicht mehr raucht

es singt der Singer

bis er nicht mehr singt

es springt der Springer

bis er nicht mehr springt

es hurt die Hure

bis sie nicht mehr hurt

es murt die Mure

bis sie nicht mehr murt

es mahnt der Mahner

bis er nicht mehr mahnt

es wahnt der Wahner

bis er nicht mehr wahnt

es nörgelt der Nörgler

bis er nicht mehr nörgelt

es wörgelt der Wörgler

bis er nicht mehr wörgelt es raubt der Rauber

bis er nicht mehr raubt

es glaubt der Glauber

bis er nichts mehr glaubt

es heizt der Heizer

bis er nicht mehr heizt

es reizt der Reizer

bis er nicht mehr reizt

es genießt der Genießer

bis er nichts mehr genießt

es beschließt der Beschließer

bis er nichts mehr beschließt

es verkehrt der Verkehrer

bis er nicht mehr verkehrt

es verehrt der Verehrer

bis er nicht mehr verehrt

es germanistelt der Germanist

bis er nicht mehr germanistelt

es slawistelt der Slawist

bis er nicht mehr slawistelt

es verlegt der Verleger

bis er nicht mehr verlegt

es erregt der Erreger

bis er nicht mehr erregt

es regiert der Regierer

bis er nicht mehr regiert

es verliert der Verlierer

bis er nicht mehr verliert

es erhebt der Erhebende

bis er nicht mehr erhebt

es lebt der Lebende

bis er nicht mehr lebt

es richtet der Richter

bis er nicht mehr richtet

es dichtet der Dichter

bis er nicht mehr dichtet. 
Abgesehen von der trotz des ironischen Untertitels mitschwingenden düsteren Andeutung von Tod und Vergänglichkeit ist der Bernhardsche Text auch wegen seiner ironisch benutzten sprachlichen Inkorrektheiten (z.B. „es raubt der Rauber", „es glaubt der Glauber") und des Gebrauchs nicht existenter Worte (z.B. „es murt die Mure“, „es wörgelt der Wörgler") nur für Leser geeignet, die im Deutschen sprachlich sicher sind. Darüber hinaus dürfte die Erwähnung von Hure, Germanist und Slawist Leser im Kindesalter überfordern. Jedoch zeigt dieser Vergleich, wie modern und nutzbar das Schema und Verfahren ist, das Michaelis - sicherlich mit einer anderen Intention - benutzt hat.

Die Gedichte in diesem Teil des Buches Zauberhut sind fast alle insofern traditioneller Art, da sie über Reime und Strophen, über ein festes Reimund Strophenschema verfügen. Sie erinnern an Reime, Sprüche, Abzählreime und Gedichte der ungarndeutschen Volksüberlieferung, sind sprachlich einfach gehalten, man kann sie mit Hilfe der Reime und des Rhythmus' leicht erlernen. Jedoch findet sich schon hier eine Ausnahme von der herkömmlichen Art des Dichtens in der Gestalt des Figurengedichtes Unsere Fahne, das sich deutlich an der Verfahrensweise der so genannten konkreten Poesie orientiert, indem der Text nicht nur die Beschreibung eines Sachverhaltes, Lebewesens oder Gegenstandes liefert, sondern der Text selber, die gedruckten Grapheme eine graphische Darstellung andeuten, wie hier der Text die Umrisse einer Fahne ergibt (ZH, S. 28):

\author{
Rot wie Schönheit \\ Rot wie Rose \\ Weiß wie Weisheit \\ Weiß wie Nelke \\ Grün wie Freiheit \\ Grün wie Knospe
}

So ist
unsre
bunte
Fahne

Dabei ist die Tradition des Figurengedichtes bis in die barocke Literatur im 17. Jahrhundert zurückzuverfolgen, wobei allerdings auffällt, dass ironischerweise in der Barockliteratur viel kompliziertere typographische Anordnungen anzutreffen sind als in der konkreten Poesie, was deutlich im Vergleich des barocken Gedichtes Sanduhr von Theodor Kornfeld (16361698, Kornfeld 1685, p. 83) mit Eugen Gomringers (1977, p. 77) berühmten Gedicht schweigen auffällt: 


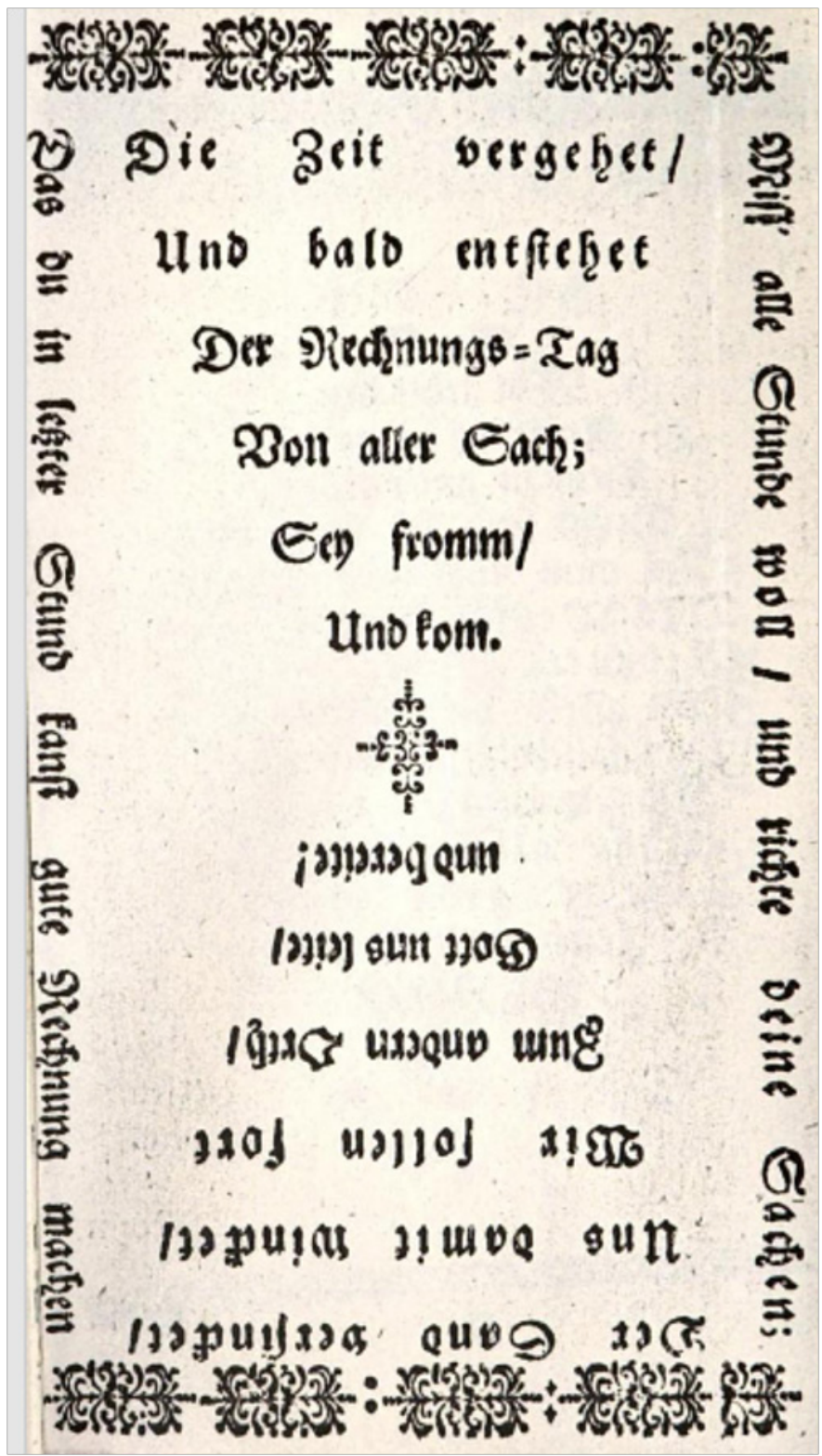


schweigen schweigen schweigen schweigen schweigen schweigen schweigen schweigen schweigen schweigen schweigen schweigen schweigen schweigen

Über den Umstand der Vermittlung neuen Wortschatzes an die Kinder ist gerade der Aspekt des Buches hervorhebenswert, dass er behutsam mit verschiedenen Formen, so auch mit modernen Formen der Literatur bekannt macht.

\subsubsection{Jahreszeiten}

Im Abschnitt Jahreszeiten finden die bereits erwähnten Anknüpfungen sowohl an die Formen der ungarndeutschen Überlieferungen als auch an die literarischen Traditionen der früheren Jahrhunderte wie Zählreime (Sieben schlimme Kameraden), Kinderlieder $(A B C)$ und konkrete Poesie (Sprühregen, Tropfen, Winter) statt. Ohne an dieser Stelle auf alle Beispiele eingehen zu können, sollen zwei Aspekte genannt werden:

1. Im Vergleich zum ersten Teil sind die hier angeführten Texte schon etwas komplizierter, sie erwarten bereits Leser aus der Oberstufe der Volksschule, wie das aus dem Titel von Liebesbrief. (Aus der 6. Klasse) auch deutlich hervorgeht.

2. Die hier benutzten Schemata der Kinderliteratur sind bis heute lebendig und sie leben im Bereich der populären Kultur weiter. Auffällig ist wie gerne in der modernen Unterhaltungsmusik die von Michaelis genutzten Schemata der Kinderliteratur aufgegriffen werden, man denke nur an das an Kinderlieder anknüpfende Lied All together now (Lennon / McCartney, 1969, 1/3) der Beatles oder das sich deutlich als Zählreim entpuppende Lied Zehn kleine Jägermeister (Rohde, Frege \& Müller, 1996, p. 18) der deutschen Rockgruppe Die Toten Hosen. Dabei greifen beide Seiten - also Michaelis (ZH, S. 44 und 39) und die Komponisten - auf die gleichen Traditionen zurück:

Michaelis:

ABC - am Morgen trink ich heißen Tee,

DEF - um neun Uhr kommt mein Freund, Detlef.

Lennon-McCartney:

A B C D - Can I bring my friend to tea?

E F G H I J - I love you

Michaelis:

Sieben schlimme Kameraden,

sie kletterten aufs Dach, 
da brach die Latte unter einem, nur sechs schrien auf: „Ach!“

Rohde / Frege / Müller:

Zehn kleine Jägermeister

rauchten einen Joint.

Den einen hat es umgehauen, da waren's nur noch neun.

Insofern kann man keinesfalls davon sprechen, dass diese Formen veraltet, ausgestorben wären und heute keine Rolle mehr spielen würden, wobei allerdings eingeräumt werden muss, im Gegensatz zu der traditionell reinen Art wie Michaelis mit diesen Formen umgeht, sind in den beiden Beispielen aus der populären Musik die Kinderliteraturschemata textlich jeweils durch einen Refrain ergänzt worden, der außerhalb des traditionellen Aufbaus steht.

\subsubsection{Zauberhut}

Der dritte Teil des Buches enthält Gedichte und Prosastücke, wobei zunächst die Gedichte folgen, wodurch sich bei der Lektüre erst einmal die Kontinuität des Lyrischen fortsetzt, doch ist dieser Teil durch das Einführen der märchenhaften Elemente anders als die vorhergehenden Teile des Buches. In diesen Gedichten erscheint ein Zauberer (Zauberhut) und immer wieder Tiere (z.B. Tierkonzert, Tierische Gewohnheiten, Theofil und Krokodil), dabei sind sie geeignet, den Kindern die Benennung der Laute näherzubringen, die die einzelnen Tiere von sich geben („Grillen zirpen, Käfer surren, / Igel schnaufen, Tauben gurren" heißt es in Tierkonzert [ZH, S. 52]), entbehren aber auch nicht einer Ironie, die für Kinder als solche erkennbar ist („Die Strauße, die Strauße, / sie trinken gerne Brause." in Tierische Gewohnheiten [ZH, S. 57). Alle Gedichte sind hier auf traditionelle Art aufgebaut und verfügen auch dementsprechend über Reime.

Den Gedichten folgen dann acht Märchen und eine Sage aus dem Jahr 1526, als in der Zeit der Türkenkriege von vor den Türken flüchtenden ungarischen Kriegern ein Schatz in der Gegend von Schomberg verborgen wird, der später nicht mehr gefunden werden kann: „Der Schatz des Königs schimmert heute noch in den Truhen des Nána-Kellers irgendwo in der Flur von Schomberg und wartet darauf, jemanden zu bereichern" (ZH, S. 97). Dieser Text ist sprachlich auf einem höherem Niveau als die anderen, weshalb er nicht zufällig am Ende des Buches angeordnet worden ist.

Den Übergang zwischen den Märchen und der Sage stellt der märchenhafte Text Die Steinschnecken von Wieland (ZH, S. 89-92) dar, in dem mit den Märchen eigenen Elementen, aber auf einen konkreten Ort in Südungarn bezogen die Entstehung der Gegend um den Jungfraugipfel bei Wieland erzählt wird, was natürlich für Leser mit Ortskenntnissen eine besonders interessante Lektüre darstellt. 
Die restlichen sieben Märchen weisen eine Reihe von Gemeinsamkeiten auf. In allen sind die auch bereits im Titel vorkommenden Hauptfiguren Tiere (Der Maulwurf und die Feldmaus, Der Elefant und die Kobra, Der Hirsch und der Wolf, Das Igelmädchen, Der Fuchs und der Hase, Der Papagei, Der Fisch und der Frosch), wobei bis auf die exotischen Beispiele Elefant, Kobra und Papagei alle anderen Tiere der heimatlichen Fauna angehören und so auf für den kindlichen Lesern Bekanntes verweisen. In den Texten geht es darum, wie bestimmte körperliche Eigenschaften (z.B. der kurze Schwanz des Hasen und der lange des Fuchses) und Verhaltensweisen (z.B. das Wühlen der Feldmäuse und Maulwürfe in der Erde, das Röhren des Hirsches, das Quaken des Frosches) der Tiere entstanden sind. Auffällig ist dabei einerseits das Fehlen jeglicher übernatürlicher Elemente und andererseits das Nichtvorhandensein der in vielen traditionellen Märchen anzutreffenden Gewalt, Missgunst und negativen Gestalten, die durch die einfache Typologie der Gattung Märchen, in der keine Differenzierung der Gestalten möglich ist, in ihrer Bösartigkeit für Kinder besonders furchteinflößend erscheinen können, weshalb häufig klassische Märchenbücher für Kinder nur bearbeitete Versionen der Texte bieten, die von den Grausamkeiten des Originals befreit worden sind. Die Michaelisschen Märchen sind in dieser Hinsicht zivilisierter und kindgerechter, sie kommen ohne die Darstellung von niederen Instinkten, Gewalt und Bösartigkeit aus.

Im Späteren hat Michaelis die Prosatexte dieses Bandes in sein zweisprachiges Buch Der verlorene Schatz / Az elveszett kincs ${ }^{6}(2008,2016)$ aufgenommen, in dem auch noch eine weitere Reihe von Sagen zu finden ist.

\subsection{Anwendung im Unterricht}

Bei der Verwendung literarischer Texte im Unterricht gibt es spezielle Regeln und Gesichtspunkte zu befolgen, bei der Benutzung von der Literatur einer nationalen Minderheit ist das nicht anders (zur Vermittlung von Sprache durch ungarndeutsche literarische Werke vgl.: Kerekes, 2019, Müller \& Balázs-Lukács, 2017). Das Buch von Josef Michaelis ist in Ungarn von den sich mit den Fragen des Deutschunterrichts Beschäftigenden mehrfach untersucht worden und es sind auch konkrete Aufgaben ausgearbeitet worden, die im Unterricht mit Hilfe der Texte von Michaelis genutzt werden können.

Ein besonders gutes Beispiel stellen die Aufgaben dar, die Éva Márkus (2007) in ihrem Band "Ene bene Tintenfass geh" zur Schul' und lerne was!" vorstellt. Alle durch die Texte von Michaelis gebotenen Möglichkeiten werden hier genutzt, so wird zum Beispiel in der das bereits zitierte Gedicht Reimschnitzerei nutzenden Aufgabe die Benennung der die einzelnen Berufe Ausübenden weggelassen und nach diesen gefragt. Das Prosamärchen Der Maulwurf und die Feldmaus wird auf die Weise zur Kontrolle der Fähigkeiten der Kinder im Textverständnis genutzt, indem die einzelnen Absätze

${ }^{6}$ Insgesamt 2.000 Exemplare. 
nicht in ihrer ursprünglichen Reihenfolge erscheinen und die Aufgabe der Kinder es ist, diese Reihenfolge zu rekonstruieren.

Ebenfalls sehr kreative Beispiele der Benutzung der Michaelisschen Texte aus Zauberhut finden sich in der mit vielen Fotografien präsentierten Beschreibung der Literaturwerkstatt mit Josef Michaelis in dem Band Ungarndeutsche Kinderliteratur in Theorie und Praxis (Klein \& Márkus, 2017).

Zauberhut von Michaelis wird auch als Perle der ungarndeutschen Kinderliteratur in Márkus (2016) erwähnt.

\section{Fazit}

Das Buch Zauberhut von Josef Michaelis ist ein Buch für Kinder und ist bis heute das erfolgreichste ungarndeutsche Buch, was angesichts seiner Vorzüge, die in der nicht aufdringlich dargebotenen Didaktik, der durchaus vorhandenen ironisch-lustigen Darstellungsweise und der seine Leser über das rein Sprachliche hinaus behutsam auf verschiedenste Arten von Literatur vorbereitenden Intention liegen, nicht überraschen kann. Nicht zufällig ist es trotz der hohen Auflagenzahl, in der es erschienen ist, vergriffen, da nicht nur die Fachleute für Didaktik und Literatur es schätzen, sondern auch seine kleinen Leser.

\section{Literatur}

Bernhard, T. (1977). Ahnenkult. Ein Gedicht für Höherstehende oder wie man sich einer hohen Aufgabe auf die kürzeste Zeit (zehn Minuten) entledigt, vollkommen gereimt und noch einmal durchgesehen von Thomas Bernhard. In Almanach auf das Jahr 1977 (pp. 21-23), Residenz.

Brantsch, I. (1994). Zauberhut. Märchen und Gedichte für Kinder. Unsere Post, 59(3), 13.

Gomringer, E. (1977). schweigen. In Gomringer, E. Konstellationen, Ideogramme, Stundenbuch. Reclam, 77.

Hermann, I. (2017). Mutatjuk, így olvas a magyar - de hogy keresnek idehaza az írók? forbes.hu, 2017.06.11. https://forbes.hu/uzlet/mutatjuk-igy-olvas-a-magyar-dehogy-keresnek-idehaza-az-irok/ (15. 05. 2020)

Kerekes, G. (2015). Ungarndeutsche Dichterportäts. Werischwarer Zeitung, 21(10), 23.

Kerekes, G. (2019). Bairische Dialektreime im Deutschunterricht. In Bordás, S. (Ed.) Módszerek és teóriák (=Methoden und TheorienI (pp. 163-176), Eötvös József Főiskolai Kiadó.

Klein, Á. \& Márkus, É. (2017, Eds.). Ungarndeutsche Kinderliteratur in Theorie und Praxis. Pécsi Tudományegyetem.

Knipf-Komlósi, E. \& Müller, M. (2018). Zwischen Vitalität und Aufgabe: Dynamische 
Aspekte in der Sprache der deutschen Minderheit in Ungarn. In Meier, J. (Ed.) Jahrbuch des Bundesinstituts für Kultur und Geschichte der Deutschen im östlichen Europa 26. (pp. 51-67), De Gruyter.

Knipf-Komlósi, E. (2011). Wandel im Wortschatz der Minderheitensprache. Am Beispiel des Deutschen in Ungarn. Franz Steiner Verlag. (= ZDL-Beihefte, Bd. 145)

Knipf-Komlósi, E. \& Müller, M. (2018). Deutsche Sprache im öffentlichen Raum einer ungarndeutschen Siedlung. In Philipp, H., Ströbl, A., Weber, B. \& Wellner, J (Eds.), Deutsch in Mittel-, Ost- und Südosteuropa: DiMOS-Füllhorn Nr. 3. (pp. 319-341), Universitätsverlag Regensburg.

Knipf-Komlósi, E. \& Müller, M. (2019). Sprachfördermaßnahmen zur Erhaltung der deutschen Sprache in Ungarn. In Ammon, U. \& Schmidt, G. (Eds.) Förderung der deutschen Sprache weltweit: Vorschläge, Ansätze und Konzepte (pp. 483-500), De Gruyter. https://doi.org/10.1515/9783110479232-030

Kornfeld, T. (1685). Ein Sand-Uhr. In Kornfeld, Theodor Selbst-lehrende alt-neue Poesie oder Vers-Kunst (p. 83).

Lennon, J. \& McCartney, P. (1969). All together now. Auf der Beatles-LP: Yellow Submarine, Seite 1 Lied Nr. 3. Apple Records PCS 7070 UK.

Márkus, É. (2007). „Ene bene Tintenfass geh'zur Schul' und lerne was!“ Eine Text- und Aufgabensammlung zur ungarndeutschen Kinderliteratur für die Studenten der NationalitätenkindergärtnerInnen- und grundschullehrerInnenbildung. Trezor Kiadó.

Márkus, É. (2009). Meine zwei Sprachen. Trezor Kiadó.

Márkus, É. (2016). A német nemzetiségi gyermekirodalom gyöngyszemei. (= Die Perlen der ungarndeutschen Kinderliteratur) In Kolosai, N. \& M. Pintér, T. (Eds.), A gyermekkultúra jelen(tốség)e (pp. 164-173). Komáromi Nyomda. http:// gyermekkultura.tok.elte.hu/docs/Gyermekkultura_ebook.pdf

Márkus, É. \& Gölcz, M. (2018). A magyarországi német nemzetiség nyelvelsajátítási szokásai. ( $=$ Der Sprachaneignungsprozess der Ungarndeutschen). Gyermeknevelés, 6(3), 56-69. https://doi.org/10.31074/201835669

Michaelis, J. (1994). Reimschnitzerei. In Michaelis, J. Zauberhut. Verband der Ungarndeutschen, 13.

Michaelis, J. (2008). Der verlorene Schatz / Az elveszett kincs. (Zweitauflage 2016)

Miskei, R. \& Müller, M. (2020). Einstellungen von jüngeren und älteren Ungarndeutschen zur deutschen Sprache und zu ihren Sprechern. In Földes, Cs. (Ed.): Beiträge zur interkulturellen Germanistik (327-343). Narr.

Müller, M. (2013). Bajor utcanevek egy magyarországi német településen (=Bairische Gassennamen in einer ungarndeutschen Siedlung). Névtani Értesitö, 35, 97-107.

Müller, M. \& Balázs-Lukács, K. (2017). Lyrikdidaktik und Mundarttexte. In Klein, Á. \& Márkus, É. (Eds.), Ungarndeutsche Kinderliteratur in Theorie und Praxis: Didaktische Handreichung für Pädagog_innen zum Unterricht der 
ungarndeutschen Nationalitätenkinderliteratur im Kindergarten und in der Primarstufe (pp. 42-49). Pécsi Tudományegyetem.

Rohde, W., Frege, A. \& Müller, H. Ch. (1996). Zehn kleine Jägermeister. Auf der Toten Hosen-CD: Opium fürs Volk 1996, Lied Nr. 18. JKP 03, EastWest - 063013829-2.

Rudolf, H. (1991). Neu für Kinder: „Zauberhut” von Josef Michaelis. Signale, 8(16). STAT = Statisztikai Tükör. Könyvkiadás 2014(=Statistischer Spiegel. Buchpublikationen 2014). Központi Statisztikai Hivatal 80(13). November 2015, 2.

Szabó, D. B. (2001). Zwischen Utopie und Realität. Deutsch-ungarische Literaturbeziehungen im Wandel. Budapester Beiträge zur Germanistik. ELTE. 


\section{Kerekes, G.}

\section{Children's literature of the Germans in Hungary: Josef Michaelis' Magic Hat}

The study deals with the most successful book of the Germans in Hungary, with Josef Michaelis' Zauberhut [Magic Hat], a book that contains poems and tales for children. It is presented in several contexts, so in the context of the Hungarian bookmarket as well as in respect of its birth and the international literary context of some poems.

Keywords: children's literature, literature of the Germans of Hungary, Josef Michaelis

Kerekes Gábor: https://orcid.org/0000-0001-9943-747X 